\title{
Complications of canine tonsillectomy by clamping technique combined with monopolar electrosurgery without suture - a retrospective study of 39 cases
}

Outi Marita Turkki ( $\square$ outi_turkki@outlook.com )

AniCura, Small Animal Hospital Bagarmossen https://orcid.org/0000-0002-8637-1800

Caroline Elisabeth Bergman

Swedish University of Agricultural Sciences: Sveriges lantbruksuniversitet

Marcel H Lee

Evidensia Södra Djursjukhuset Kungens Kurva

Odd Viking Höglund

Swedish University of Agricultural Sciences: Sveriges lantbruksuniversitet

\section{Research Article}

Keywords: Canine tonsillectomy, Dog tonsillectomy, Tonsillectomy complication, Tonsillar bleeding, Tonsillar haemorrhage, Tonsillectomy technique

Posted Date: February 21st, 2022

DOI: https://doi.org/10.21203/rs.3.rs-1355092/v1

License: (9) This work is licensed under a Creative Commons Attribution 4.0 International License. Read Full License 


\section{Abstract}

Background: Canine tonsillectomy is performed due to acute or chronic tonsillitis, neoplasia, trauma or occasionally brachycephalic obstructive airway syndrome. Several tonsillectomy techniques are used but information about surgical complications is scarce. This retrospective study of patient records at the University Animal Hospital aimed to investigate complications related to canine tonsillectomy performed by 20-minute clamping combined with monopolar electrosurgery.

Inclusion criteria were bilateral tonsillectomy performed with "20-minute clamping technique combined with monopolar electrosurgery without suture or ligation". Exclusion criteria were unilateral tonsillectomy, tonsillar neoplasia, additional surgical procedures other than tonsillectomy and cases where sutures were used initially, cases where tissue sealing only relied on electrosurgery devices or when the technique was unspecified. The search of the patient records of the University Animal Hospital included a 10-year period. Complications that required additional anaesthesia were defined as major complications. Minor complications were handled during surgery or postoperatively without surgical intervention.

Results: Of 39 dogs that fulfilled the inclusion criteria, 11 dogs had one complication and 1 dog had two complications. Altogether, of the 12 complications, 2 were classified as major complications and 10 as minor.

The most frequent complication was bleeding from the surgical site; 10 dogs had one incidence of bleeding and 1 dog bled twice, both intra- and postoperatively. Of these 11 dogs, 7 were intraoperative bleedings and 4 were postoperative. The two dogs with major complications were re-anaesthetized due to post-surgical bleeding. No lethal complications occurred and all dogs survived to discharge.

Conclusions: Complications such as bleeding are common during and after surgery for tonsillectomy using a "20-minute clamping technique combined with monopolar electrosurgery without suture". This tonsillectomy technique is not without risk and revision surgery may be necessary.

\section{Background}

Tonsillectomy in dogs is indicated for management of acute or chronic tonsillitis, trauma, neoplasia and occasionally with brachycephalic obstructive airway syndrome [1,2]. Different surgical techniques are described in veterinary medical textbooks $[1,3,4]$. The tradition is to combine a short clamping time with suture or manual ligation and mucosal suture or electrosurgical tissue sealing is recommended. The complication rate after tonsillectomy in dogs is suggested to be low [1] the need to re-anaesthetize is rare [3] but evidence is scarce [4]. The technique of forceps clamped across the tonsillar base for 20 minutes, often without complementing with suture or ligation, is widely used in Sweden. However, haemostasis primarily relies on crushed tissue, and its efficacy and related complications are not reported.

A study by Belch et al. [5] evaluated the effectiveness of a vessel-sealing device with bipolar technique, the short 5-mm LigaSure device (Covidien Inc., USA) and compared it to a standard technique with 
tonsillectomy forceps (Veterinary Instrumentation, Sheffield, UK) combined with the Parker-Kerr suture in 20 dogs. The techniques were compared on the same dog, one technique on each side of the larynx. In that study, all dogs were diagnosed and operated for brachycephalic obstructive airway syndrome (BOAS) [5]. When the LigaSure device was used ( $n=20$ tonsils) no additional sutures were needed for haemostasis. However, in one dog the LigaSure device was reapplied to control bleeding. With the standard Parker-Kerr technique, in 8 of 20 tonsils an additional suture was needed due to bleeding. Additionally, in three dogs the tonsillectomy clamp slipped after transection, and in one dog the clamp technique was abandoned due to limited space and therefore the crypt was sutured without clamp. Belch et al. reported no postoperative bleeding [5].

Cook et al. [6] used the bipolar Small Jaw LigaSure Instrument (Covidien Inc., USA) and all 22 BOAS related tonsillectomies were completed without intra- or postoperative bleeding complications. In a study by Eesa [7] which included 11 dogs, a traditional ligation technique was compared to an electrocautery technique with or without use of an endoscope. One minor intraoperative bleeding was reported when traditional ligation was used [7].

The objective of this retrospective study was to investigate the incidence of intra- and postoperative complications in canine tonsillectomy performed with 20-minute clamping technique combined with monopolar electrosurgery without sutures by using the digital medical record database at the University Animal Hospital, Swedish University of Agricultural Sciences, Uppsala (SLU). We hypothesized that the complication rate would be low and that the most common complication would be bleeding.

\section{Methods}

\section{Data collection}

The digital medical record database at SLU was screened for canine cases where tonsillectomy was performed during the period from Jan 1st, 2007 to Dec 31st, 2016. Retrieval of patient data was done six months after the most recent surgery. Inclusion criteria was bilateral tonsillectomy performed by using 20-minute clamping combined with monopolar electrosurgery, without suture or ligation. Exclusion criteria were unilateral tonsillectomy, tonsillar neoplasia, additional surgical procedure other than tonsillectomy, cases where sutures were used initially, and cases where tissue sealing only relied on electrosurgery devices or if the technique was unspecified.

The following information was collected from the digital medical records: breed, age, body weight, sex and neuter status, indication for surgery, surgical details and complications during and post-surgery up to one week [8]. The surgical details included duration of clamping time, recorded complications as well as treatment interventions during and after surgery. Body weight, age and clamping time were presented as median and interquartile range (IQR).

\section{Surgical technique}


The studied surgical technique, here called "20-minute clamping technique combined with monopolar electrosurgery without suture", is a modified standard technique without suture where tonsillar tissue is grasped with forceps and clamps are placed across the tonsillar base for 20 minutes before transection of tissue. The transection is done with monopolar diathermy or scissors combined with monopolar diathermy.

\section{Complications}

Minor complications were defined as complication that were handled intraoperatively or postoperatively and did not require a new surgical intervention. Major complications were defined as complications that required re-anaesthetizing the patient for revision surgery.

\section{Results}

\section{Distribution of cases and characteristics}

Sixty-six cases of canine tonsillectomies were identified in the dataset. The following cases were excluded: unilateral tonsillectomy after trauma $(n=1)$, squamous cell carcinoma $(n=4)$, additional oropharyngeal procedures $(n=11)$ and other surgeries with vaguely described or unspecified techniques $(n=11)$.

Thirty-nine dogs fulfilled the inclusion criteria, 23 males and 16 females, of which 3 males and 1 female were gonadectomized. The median age of dogs was 2.9 years (IQR 1.6-5.7) at time of surgery.

The three most common breeds were Standard Poodle $(n=4)$, Irish Setter $(n=3)$, Dutch hound $(n=3)$, three of the dogs were mixed breeds.

Median body weight was $18.2 \mathrm{~kg}$ (IQR 9.0-28.2): 14 dogs weighed less than $10 \mathrm{~kg}, 17$ dogs weighed 10 to $30 \mathrm{~kg}$ and 8 dogs weighed more than $30 \mathrm{~kg}$. Median clamping time was 21 minutes (IQR 20-30).

\section{Indication for surgery}

The main indication for tonsillectomies was chronic tonsillitis $(n=36)$, followed by acute tonsillitis $(n=2)$ and acute trauma with ongoing history of chronic tonsillitis $(n=1)$.

\section{Complications}

In total, 12 incidences of complications were found; 11 of bleeding and 1 of coughing with signs of laryngeal pain after surgery. One dog was recorded twice for both intra- and postoperative bleeding. Of these 12 incidences, 7 were during surgery and 5 postoperatively (Table 1). 
Table 1

Minor and major surgical complications after 39 canine tonsillectomies

\begin{tabular}{|llll|}
\hline Complication & Total & Minor & Major \\
\hline Total & 12 & 10 & 2 \\
\hline Intraoperative haemorrhage & 7 & $7 *$ & - \\
\hline Postoperative haemorrhage - conservative treatment & 2 & $2 *$ & \\
\hline Postoperative haemorrhage - surgery required & 2 & - & 2 \\
\hline Signs of pharyngeal pain and coughing at day four & 1 & 1 & - \\
\hline $\begin{array}{l}* \text { One dog belonged to both groups; intraoperative bleeding and postoperative bleeding with } \\
\text { conservative treatment }\end{array}$ & & & \\
\hline
\end{tabular}

Table 1

\section{Minor complications}

Minor complications were reported in 10 dogs, of which 7 consisted of intraoperative bleeding that was controlled during surgery. Surgical control of intraoperative bleeding was achieved by suturing the tonsillar fold $(n=4)$ over the empty crypt with a simple interrupted or continuous suture pattern or a combination of manual ligation and tonsillar fold suturing $(n=2)$ or the use of monopolar diathermy $(n=$ 1). Poliglecaprone 25 (Monocryl, Ethicon) was used as a ligation or suture material in all cases.

Three dogs had minor postoperative complications. Two dogs with postoperative oral bleeding during hospitalisation were treated conservatively without surgical intervention. In both cases with oral bleeding, the bleeding stopped and haemostasis was verified. In one of these dogs bleeding was observed 10 minutes after recovery from anaesthesia and diminished within one hour without surgical intervention. The dog was treated with a single dose of $10 \mathrm{mg} / \mathrm{kg}$ iv. tranexamic acid (Cyklokapron $\circledast$, Pfizer). The other dog showed bloody salivation directly after anaesthesia, but signs of oral bleeding disappeared after a few hours without any treatment. A third dog with minor complications was coughing with signs of laryngeal pain on the fourth day after surgery. The dog was presented on a recheck and treated with nonsteroid anti-inflammatory medication $2 \mathrm{mg} / \mathrm{kg}$ twice a day po. (Rimadyl® vet., Zoetis) for five days. Laryngeal signs resolved.

\section{Major complications}

Major complications occurred in two dogs with haemorrhage from the tonsillar vessels. In one of them, haemostasis was achieved by a combination of clamping for 20 minutes and monopolar diathermy. In the other dog that was re-anesthetized, the tonsillar fold was sutured and the vessel was ligated. Both dogs recovered uneventfully.

\section{Discussion}


Complications after canine tonsillectomy without suturing were investigated from an intra- and postoperative perspective, and the most common complication was bleeding from the surgical site. Although no fatal complications were found in the study population, the identification of 10 minor and 2 major complications out of 39 cases raises concerns about the use of the technique.

Comparisons between earlier studies and the present study have to be done cautiously due to different patient groups, diagnosis, differences in duration of ongoing pathology and clinical signs. Both Cook et al. [6] and Belch et al. [5] performed tonsillectomy in brachycephalic dogs with obstructive airway syndrome amongst other surgical interventions such as staphylectomy, laryngeal sacculectomy and rhinoplasty. The most common complications, described in both studies were coughing, regurgitation, inappetence and laryngeal oedema. There is an increased risk of complications in brachycephalic dogs $[9,10]$. In the present study, BOAS surgery cases were excluded.

Veterinary medical publications report a low risk for bleeding post tonsillectomy when using vessel sealing devices. This study found a higher incidence of bleeding, 11 out of 39 dogs, compared to previous studies using energy-based vessel sealing devices $[5,6]$. In the study by Belch et al. [5] the LigaSure device had to be reapplied once in 20 tonsils. Cook et al. [6] also used the LigaSure device and reported no bleeding in 22 dogs. The present technique, with over 20 minutes of clamping of the tonsillar base, indicated a significantly longer duration of surgery compared to Belch's use of LigaSure with less than one minute for seal and cut [5]. The present study involved electrosurgery but use of the technique was not standardised, which is different compared to use of energy based vessel sealing devices such as LigaSure. Shorter surgical time and less bleeding are beneficial for the patient. Considering the results of the present study and the available data from other studies, a preference for the use of standardised energy-based techniques, vessel sealing devices, may be suggested for tonsillectomy. However, a randomized prospective study to evaluate advantages and drawbacks of each technique should be done.

Tonsillectomy is a common surgical procedure in humans. Considering species differences, comparison of human studies with veterinary medical studies should be done cautiously. Furthermore, surgical facilities and perioperative care may also differ substantially. Large retrospective studies of human data reported a wide range of complications such as bleeding from the surgical site, infections, other surgical site complications, dehydration, upper respiratory obstruction, postoperative pain and inadequate oral nutrition as commonly recognized complications [11-13]. A study by Seshamani et al. [14] reported postoperative complication rate for human tonsillectomies to be $20 \%$, mainly haemorrhage, dehydration and pain, whereas another study by Chen et al. [11] reported a lower rate, $1.2 \%$, mainly infections.

In several reported human case series, the rate of post tonsillectomy bleeding ranged from $2.0-7.0 \%$ [12, 14-21]. However, definitions varied slightly. Sarny et al. reported higher rates of post tonsillectomy bleeding, from $14-16 \%$ in four different studies [22-25]. There is conflicting evidence about surgical technique and equipment in studies evaluating the risk for post tonsillectomy bleeding. A meta-analysis by Francis et al. [18] reported that frequency of post tonsillectomy bleeding across different techniques did not differ. Krishna et al. [26] reported benefits for monopolar surgery but two other studies described 
higher risk of bleeding for "hot" tonsillectomy techniques, when diathermy was used for dissection, compared to traditional "cold steel" [21, 27].

The present study had several limitations. Surgeries were performed by different surgical teams and reports of complications were therefore inconsistent. The decision to treat intra- and postoperative bleeding was at the discretion of the surgeon and may have differed between surgeons, which influenced the classification of a complication. Furthermore, an incident may not be regarded as a complication, but rather an expected sequela of surgery. Other limitations were the small number of dogs, weight variation, age and type of dogs. An additional limitation was the lack of long-term follow-up; owners of the dogs were not contacted and potential late complications may have been treated at another clinic.

This study was not designed to evaluate or compare safety of different techniques. Due to the involvement of several different surgeons and the retrospective nature of the study, the results of the present study should be used cautiously in recommendation of any particular surgical technique. A prospective randomized study comparing several techniques is needed.

\section{Conclusions}

Most of the intra- and postoperative complications were classified as minor and were easily managed. Minor complications such as bleeding were common with the use of the presented "20-minute clamping technique combined with monopolar electrosurgery without suture or ligation". Revision surgery may be needed and sometimes this surgery may be urgent. Although the assessed technique has not been compared with another technique, combining it with suturing should be considered to reduce risk of bleeding. Alternatively, the surgeon should consider using a standardized instrument such as a bipolar vessel sealing device to reduce risk of complications.

\section{Abbreviations}

\section{BOAS}

brachycephalic obstructive airway syndrome

$\mathrm{n}$

number

IQR

interquartile range

iv.

intra venous

po.

per oral

\section{Declarations}




\section{Ethics approval and consent to participate}

This study did not require official or institutional ethical approval due to of the study's retrospective design. The digital medical record database was available, and no permission was needed.

\section{Consent for publication}

Not applicable.

\section{Availability of data and materials}

Corresponding author can be requested for detailed data used for the study.

\section{Competing interests}

\section{The authors declare that they have no competing interests.}

\section{Funding}

No grants were involved in this study.

\section{Authors' contributions}

$\mathrm{OVH}$ designed the retrospective study and organized the collection of the study material with $\mathrm{CB}$. OT summarised the data and drafted the manuscript. All authors collaborated in discussing the results with interpretation of the results and finishing the final draft of the manuscript. All authors read and approved the final manuscript.

\section{Acknowledgements}

\section{Not applicable.}

\section{Authors' information}

OMT is resident of European College of Veterinary Surgeons (ECVS) and this publication is a part of her residency program. CB is an assistant professor of surgery at Department of Clinical Sciences, Swedish University of Agricultural Sciences, SLU, Uppsala, Sweden. MHL is ECVS Diplomate and clinical supervisor for OMT's ECSV residency program. OVH is associate professor of surgery at Department of Clinical Sciences, Swedish University of Agricultural Sciences, SLU, Uppsala, Sweden. 


\section{References}

1. Anderson GM. Soft tissues of the oral cavity. In: Johnston SA, Tobias KM, editors. Veterinary surgery: small animal. 2nd edition. St. Louis, Missouri: Elsevier Saunders; 2018. p. 1650-2.

2. Todoroff RJ, Brodey RS. Oral and pharyngeal neoplasia in the dog: a retrospective survey of 361 cases. J Am Vet Med Assoc. 1979;175:567-71.

3. Dulisch ML. The Tonsils. In: Slatter D, editor. Textbook of small animal surgery. 3rd edition. Philadelphia, PA: Saunders; 2003. p. 1079-83.

4. Radlinsky MG. Surgery of the digestive system. In: Welch Fossum T, editor. Small animal surgery. 4th edition. St. Louis, Missouri: Elsevier Inc.; 2013. p. 395-6.

5. Belch A, Matiasovic M, Rasotto R, Demetriou J. Comparison of the use of LigaSure versus a standard technique for tonsillectomy in dogs. Vet Rec. 2017;180:196.

6. Cook DA, Moses PA, Mackie JT. Clinical effects of the use of a bipolar vessel sealing device for soft palate resection and tonsillectomy in dogs, with histological assessment of resected tonsillar tissue. Aust Vet J. 2015;93:445-51.

7. Eesa MJ. Comparative study of three methods of tonsillectomy in dogs. Basrah J Vet Res. 2007;6:18-24.

8. Bowyer AJ, Royse CF. Postoperative recovery and outcomes - What are we measuring and for whom? Anaesthesia. 2016;71:72-7.

9. Gruenheid M, Aarnes TK, McLoughlin MA, Simpson EM, Mathys DA, Mollenkopf DF, et al. Risk of anesthesia-related complications in brachycephalic dogs. J Am Vet Med Assoc. 2018;253:301-6.

10. Downing F, Gibson S. Anaesthesia of brachycephalic dogs. J Small Anim Pract. 2018;59:725-33.

11. Chen MM, Roman SA, Sosa JA, Judson BL, KA C, MJ H, et al. Safety of adult tonsillectomy: A population-level analysis of 5968 patients. JAMA Otolaryngol Neck Surg. 2014;140:1-25.

12. Galindo Torres BP, De Miguel García F, Whyte Orozco J. Tonsillectomy in adults: Analysis of indications and complications. Auris Nasus Larynx. 2018;45:517-21.

13. Goldstein NA, Armfield DR, Kingsley LA, Borland LM, Allen GC, Christopher Post J. Postoperative complications after tonsillectomy and adenoidectomy in children with Down syndrome. Arch Otolaryngol Head Neck Surg. 1998;124:171-6.

14. Seshamani M, Vogtmann E, Gatwood J, Gibson TB, Scanlon D. Prevalence of complications from adult tonsillectomy and impact on health care expenditures. Otolaryngol Head Neck Surg. 2014;150:574-81.

15. Bhattacharyya N. Healthcare disparities in revisits for complications after adult tonsillectomy. Am J Otolaryngol. 2015;36:249-53.

16. Windfuhr JP. Specified data for tonsil surgery in Germany. GMS Curr Top Otorhinolaryngol Head Neck Surg. 2016;15:1-29. 
17. Chen MM, Roman SA, Sosa JA, Judson BL. Safety of adult tonsillectomy: A population-level analysis of 5968 patients. JAMA Otolaryngol - Head Neck Surg. 2014;140:197-202.

18. Francis DO, Fonnesbeck C, Sathe N, McPheeters M, Krishnaswami S, Chinnadurai S. Postoperative bleeding and associated utilization following tonsillectomy in children. Otolaryngol Head Neck Surg. 2017;156:442-55.

19. Hoddeson EK, Gourin CG. Adult tonsillectomy: current indications and outcomes. Otolaryngol Head Neck Surg. 2009;140:19-22.

20. Shay S, Shapiro NL, Bhattacharyya N. Revisit rates and diagnoses following pediatric tonsillectomy in a large multistate population. Laryngoscope. 2015;125:457-61.

21. Lowe D, van der Meulen J, Cromwell D, Lewsey J, Copley L, Browne J, et al. Key messages from the National Prospective Tonsillectomy Audit. Laryngoscope. 2007;117:717-24.

22. Sarny S, Ossimitz G, Habermann W, Stammberger H. [Austrian tonsil study part 3: Surgical technique and postoperative haemorrhage after tonsillectomy]. Laryngorhinootologie. 2013;92:92-6.

23. Sarny S, Habermann W, Ossimitz G, Stammberger H. [The Austrian Tonsil Study 2010 - Part 2: Postoperative haemorrhage]. Laryngorhinootologie. 2012;91:98-102.

24. Sarny S, Ossimitz G, Habermann W, Stammberger H. Hemorrhage following tonsil surgery: a multicenter prospective study. Laryngoscope. 2011;121:2553-60.

25. Sarny S, Habermann W, Ossimitz G, Stammberger $H$. What lessons can be learned from the Austrian events? ORL J Otorhinolaryngol Relat Spec. 2013;75:175-81.

26. Krishna P, LaPage MJ, Hughes LF, Lin SY. Current practice patterns in tonsillectomy and perioperative care. Int J Pediatr Otorhinolaryngol. 2004;68:779-84.

27. Schmidt R, Herzog A, Cook S, O'Reilly R, Deutsch E, Reilly J. Complications of tonsillectomy: a comparison of techniques. Arch Otolaryngol Head Neck Surg. 2007;133:925-8. 